\title{
Default mode network dissociation in depressive and anxiety states
}

\author{
Joana Fernandes Coutinho ${ }^{1} \cdot$ Sara Veiga Fernandesl ${ }^{1} \cdot$ José Miguel Soares $^{2}$. \\ Liliana Maia $^{1}$ - Óscar Filipe Gonçalves ${ }^{1,3,4,5}$ - Adriana Sampaio ${ }^{1}$
}

Published online: 26 March 2015

(C) Springer Science+Business Media New York 2015

\begin{abstract}
The resting state brain networks, particularly the Default Mode Network (DMN), have been found to be altered in several psychopathological conditions such as depression and anxiety. In this study we hypothesized that cortical areas of the DMN, particularly the anterior regions - medial
\end{abstract}

Joana Fernandes Coutinho holds a Ph.D. degree in Clinical Psychology, University of Minho.

Sara Veiga Fernandes holds a Master degree in Psychology, University of Minho.

José Miguel Soares holds a Ph.D. degree in Health Sciences, University of Minho.

Liliana Maia holds a Master degree in Biomedical Engineering, University of Minho.

Óscar Filipe Gonçalves holds a P.hD. degree in Counseling and Consulting Psyhcology, University of Massachusetts, Amherst.

Adriana Sampaio holds a Ph.D. degree in Clinical Psychology, University of Minho.

Joana Fernandes Coutinho

joanafpc@gmail.com

1 Neuropsychophysiology Lab, CIPsi, School of Psychology, University of Minho, Braga, Portugal

2 Life and Health Sciences Research Institute and ICVS-3Bs PT Government Associate Laboratory, Universityof Minho, Braga, Portugal

3 Department of Counseling and Applied Educational Psychology, Bouvé College of Health Sciences, Northeastern University, Boston, MA, USA

4 Spaulding Neuromodulation Center, Department of Physical Medicine and Rehabilitation, Spaulding Rehabilitation Hospital, Boston, MA, USA

5 Massachusetts General Hospital, Harvard Medical School, Boston, MA, USA prefrontal cortex and anterior cingulate cortex - would show an increased functional connectivity associated with both anxiety and depression. Twenty-four healthy participants were assessed using Hamilton Depression and Anxiety Rating Scales and completed a resting-state functional magnetic resonance imaging scan. Multiple regression was performed in order to identify which areas of the DMN were associated with anxiety and depression scores. We found that the functional connectivity of the anterior portions of DMN, involved in self-referential and emotional processes, was positively correlated with anxiety and depression scores, whereas posterior areas of the DMN, involved in episodic memory and perceptual processing were negatively correlated with anxiety and depression scores. The dissociation between anterior and posterior cortical midline regions, raises the possibility of a functional specialization within the DMN in terms of selfreferential tasks and contributes to the understanding of the cognitive and affective alterations in depressive and anxiety states.

Keywords Default mode network · Anterior-posterior dissociation $\cdot$ Depressive states $\cdot$ Anxiety states

\section{Background}

Cognitive neuroscience has contributed to the advancement of understanding of psychological disorders and their symptoms by systematically investigating the presence of possible alterations in brain structure and function in clinical populations when compared to healthy controls.

Functional studies of the neural networks that were identified during the brain's resting state and its relationship with different neuropsychiatry disorders have been growing in the last decades. Among the existing resting state networks, the 
Default Mode Network (DMN), has been the object of particular attention due to its involvement in self-referential processes often affected in clinical conditions (e.g., depression; anxiety) such as evaluating the salience of internal and external cues, remembering the past, and planning the future. The significance of the DMN for contemporary models of mental illness has been empirically supported by findings on alterations of this network associated with different neuropsychiatry disorders (e.g., Buckner et al. 2008; Calhoun et al. 2009; Fox and Raichle 2007; Garrity et al. 2007; Greicius et al. 2007; Greicius et al. 2003; Raichle et al. 2001; Sorg et al. 2007; Whitfield-Gabrieli and Ford 2012). There is an increasing number of studies focused on DMN alterations in a wide range of clinical conditions as well as in normal psychological states with a diversity of methodological approaches: DMN connectivity at rest; transition from rest to externally-oriented tasks; alterations in the level of activity of specific regions of interest of the DMN either at rest or during specific emotional tasks (Berman et al. 2011; Kennedy and Courchesne 2008; Liao et al. 2010; Öngür et al. 2010; Uddin et al. 2008; Zhao et al. 2007; Zhou et al. 2007). In what follows we will report studies looking at DMN functional connectivity in depression and anxiety (e. g., Zhou et al. 2010; Zhao et al. 2007; Zhu et al. 2012). A relatively large number of studies have found an increase in DMN resting-state connectivity in depressed subjects (e. g., Zhou et al. 2010; Hamilton et al. 2011). Importantly, studies looking at the activity of DMN anatomical areas during different emotional tasks showed that DMN alterations in depression are associated with higher levels of maladaptive rumination and negative self-attribution (Grimm et al. 2009; Kross et al. 2009; Hamilton et al. 2011), suggesting that DMN alterations may modulate the mood alterations. Depressive symptomatology is characterized by feelings of sadness and anhedonia, vegetative symptoms and can cause related changes in appetite, weight, sleep, energy, and sexual activity (APA 2013). In addition, depressive states include cognitive symptoms such as rumination, feelings of guilt, and difficulty in decision making (Beck 1976; Mor and Winquist 2002).

An important empirical finding is that the anterior portions of the DMN seem to be those with more significant alterations in depressed patients (Greicius et al. 2007; Northoff et al. 2006; Zhu et al. 2012), pointing towards the possibility of a dissociation between the anterior and posterior parts of the DMN. For example, Zhu et al. (2012) found that depressed patients showed increased resting functional connectivity in the medial prefrontal cortex (MPFC), ventral anterior cingulate cortex (ACC), and medial orbitofrontal (OBF) cortex, but decreased functional connectivity in the posterior cingulate cortex (PCC) and adjacent precuneus and angular gyrus. Interestingly the anterior medial cortex regions correlated positively with rumination score, while the decreased functional connectivity in the posterior medial cortex correlated negatively with over general autobiographical memory score. In the same direction, Greicius et al. (2007) suggested that persistent, emotionally laden, self-reflective tendencies like rumination in depressed persons might generate increased activity in medial prefrontal portions of the DMN, whereas episodic memory retrieval impairments might lead to reduced activity in the more posterior regions of the network.

The same type of DMN alterations seem to apply to anxiety, which very often is comorbid to depression (Norman et al. 1996). In fact, $85 \%$ of patients with depression also experience significant symptoms of anxiety. In the same way, comorbid depression occurs in up to $90 \%$ of the patients with anxiety disorders (Gorman 1996). Like depression, anxiety disorders are associated with an attentional bias and selffocused attention (Clark and Wells 1995). Worry and rumination lead to the perpetuation of negative affect and thought with negative effects on interpersonal functioning and physical health (Watkins 2008). Similarly to depression, studies looking at DMN alterations in anxiety have looked at both, DMN patterns of connectivity at rest and DMN activation during functional tasks. For example Zhao et al. (2007) in a study that looked at the functional activation of the DMN with anxiety patients found evidence for a an anterior-posterior DMN dissociation (i.e., reduced deactivation of the medial prefrontal cortex - MPFC; increased deactivation of the posterior cingulate cortex - PCC) while listening to threat-related words as compared to a resting condition. Interestingly, this anterior-posterior DMN dissociation is consistent with studies reporting higher levels of activation at rest of the MPFC and adjacent ACC in disorders such as social anxiety (Liao et al. 2010) simple phobias (Clark and Wells 1995), as well as anxiety states in healthy controls (Chua et al. 1999; Wang et al. 2008; Kimbrell et al. 1999; Cameron et al. 2000). In an opposite direction, the posterior DMN areas seem to be inversely correlated with anxiety. Osuch et al. (2000) found a negative association between anxiety symptoms in healthy individuals and posterior areas like the left fusiform, left superior temporal, and left angular gyrus. In the same direction, a recent study by Sampaio et al. (2013) found that the personality trait of neuroticism (a predisposing factor to anxiety and depression states) was negatively correlated with the functional connectivity of DMN's parietal regions.

Taken together, the results of the abovementioned studies suggest that certain regions of the DMN, rather than the DMN as a whole, may be particularly involved in the maladaptive self-referential thought that characterizes both depression and anxiety. Therefore, the main goal of this study was to analyze the relationship between the functional connectivity of the DMN and depressive and anxiety states. The sample used in this study is composed by healthy individuals with no clinically significant symptoms of depression and anxiety. This option was based on evidence for the continuity between 
depression or anxiety in healthy and clinical populations and is in line with the RDoC framework included in the new strategic plan of the NIMH (Insel et al., 2010). According to the official statement of the RDoC framework: "To clarify the underlying causes of mental disorders, it will be necessary to define, measure, and link basic biological and behavioral components of normal and abnormal functioning". Consistent with this assumption, there is increasing empirical evidence that various forms of mental disorders result from alterations in normal functioning (Buckholtz \& Meyer-Lindenberg, 2012). Moreover there is evidence that regions such as the MPFC involved in depression, are equally activated during self-referential processing by both patients and healthy subjects. Additionally, there is evidence for an increased activation of the same regions when healthy subjects, with vulnerability to depression, are confronted with negative stimuli (Lemogne et al. 2009). This may suggest that the alterations in DMN frontal midline regions may be associated with current mood states prodromic of major depression episodes.

In this study, we hypothesize that individuals with higher scores in depression and anxiety will present an increased functional connectivity of the DMN, particularly in the anterior portions of this network such as the MPFC and ACC. On the contrary, we expect posterior areas of the DMN such as the PCC, precuneus and parietal areas to be negatively associated with depression and anxiety. In sum, anxiety and depression symptoms may be associated with a DMN frontal posterior dissociation.

\section{Methods}

\section{Participants}

Twenty-four healthy adults (9 men and 15 women) with a mean age of 24.13 (S.D=1.33) ranging from 20 to 27 years participated in this study. Participants were all Caucasian and university students. Participants were recruited by informal advertising of the study and had no past or current depression or anxiety disorders. The study was approved by the Institutional Review Board of the University of Minho and by the Human Ethics Committee of S. Marcos Hospital, in which data was collected. The procedures complied with the principles expressed in the Declaration of Helsinki. Exclusion criteria for the current sample were as follows: (1) the presence of any major medical or neurological disorder; (2) dependency or abuse in the past year of alcohol and/or drugs; (3) general MR contraindications. The study goals and tests were explained to all participants and all gave informed written consent. After signing the written informed consent, all the participants responded to a laterality test: the Edinburgh Handedness Inventory (Oldfield 1971). The participants underwent a session of clinical assessment to evaluate the presence and severity of depression and anxiety symptoms followed by an fMRI scanning session.

\section{Psychological assessment}

- Hamilton Depression Rating Scale (HDRS, M. Hamilton 1967) - This instrument is a clinician rated scale that assesses the severity of the depression by probing mood, feelings of guilt, suicide ideation, insomnia, weight loss, and somatic symptoms. The scale consists of 17 items defined by a series of symptoms which are scored on a scale of 0 (not present) to 4 (severe), with a total score range of $0-68 . \leq 7$ indicates normal range; $8-15$ indicates minor depression and $\geq 16$ major depression. The mean score of the participants in our sample was $4.59(\mathrm{SD}=3.2)$, which is below the cutoff of 16 that indicates the presence of clinical depression. The HDRS was administered by a trained clinician (one of the co-authors) in the format of structured interview.

- Hamilton Anxiety Rating Scale (HARS, M. Hamilton 1959)- The HARS is also a clinician-rated scale that measures the severity of both psychological (mental agitation and psychological distress) and somatic (physical complaints related to anxiety) anxiety symptoms. The scale consists of 14 items, defined by a series of symptoms which are scored on a scale of 0 (not present) to 4 (severe), with a total score range of $0-56$, where $<17$ indicates mild severity, 18-24 mild to moderate severity and $>24$ moderate to severe. In this scale the mean score of the participants was $5.75(\mathrm{SD}=5.95)$, which also indicates the absence of clinical anxiety in this sample. The HARS was administered by a trained clinician (one of the co-authors) in the format of structured interview.

\section{Functional MRI acquisition}

A 5 min resting-state $\mathrm{fMRI}$ scan was acquired using a clinically approved Siemens Magnetom Avanto 1.5 T (Siemens Medical Solutions, Erlangen, Germany) scanner. Functional images were obtained axially using a gradient- echo echo-planar imaging (EPI) BOLD fMRI acquisition sequence with the following imaging parameters: repetition time $=3000 \mathrm{msec}$; echo time $=50 \mathrm{msec}$; flip angle $=90^{\circ}$; field of view $=220 \mathrm{~mm}$; matrix $=64 \times 64$; in plane spatial resolution $=3.4 \times 3.4 \mathrm{~mm}^{2}, 100$ volumes, 30 interleaved slices and slice thickness $=5 \mathrm{~mm}$. Participants were instructed to keep their eyes closed and to remain awake doing nothing in particular during the acquisition.

\section{Image processing}

Prior to further processing and analysis, all the images were inspected to confirm that they were not affected by critical head motion and that participants had no brain lesions. All imaging preprocessing steps were conducted using DPARSF-Advanced (Chao-Gan and Yu-Feng 2010). To achieve signal stabilization and allow subjects to adjust to the scanner noise, the first 5 
volumes (15 s) were discarded. Functional MRI data were corrected for errors in slice timing, using first slice as reference. Images were realigned to the mean image to correct head motion with a six-parameter rigid-body spatial transformation. All subjects displayed head motion less than $2 \mathrm{~mm}$ in translation or $2^{\circ}$ in rotation. Images were spatially normalized to the MNI (Montreal Neurological Institute) standard coordinate system using SPM8 EPI template. Spatial smoothing using a fullwidth Gaussian Kernel at half maximum, $6 \mathrm{~mm}$ was performed to reduce noise without reducing valid activation. The linear trend of time courses were removed and the images were bandpass filtered $(0.01-0.08 \mathrm{~Hz})$. Band-pass filtering was used due to its main advantages when using ICA for resting state analysis: it minimizes the influence of both high and low frequency noise such as MRI drift, breathing and pulsation artifact, without leading to independence loss (Pignat et al. 2013). Moreover this filter was used because resting state networks are typically observed in the range of frequencies between 0.01 and 0.08 .

\section{Independent component analysis}

To analyze the functional networks active in the task-free BOLD sequence, spatial independent component analysis ICA analysis was performed using the Group ICA 2.0 of fMRI Toolbox (GIFT, http://www.icatb.sourceforge.net) (Calhoun et al. 2009; Correa et al. 2005). The ICA analysis consists in extracting the individual spatial independent maps and their related time courses (Beckmann et al. 2005). The reduction of dimensionality of the functional data and computational load was performed with Principal Component Analysis (PCA). The number of Independent Components estimated was 20 for each subject. ICA calculation were then carried out using the iterative Infomax algorithm. The ICASSO tool was used to control the ICA reliability. Twenty computational runs were made on the dataset, during which the components were being recomputed and compared across runs and the robustness of the results was ensured (Himberg et al. 2004). The independent components were obtained and each voxel of the spatial map was expressed as a t-statistic map, which was finally converted to a z-statistic that characterizes the degree of correlation of the voxel signal with the component time course, providing indirectly a degree of functional connectivity within the network (Greicius et al. 2007; Bartels and Zeki 2005). The 20 components estimated for each subject represent the independent sources of variability in the resting state data, thus overall they represent the functional networks active in the resting state: one component represents the DMN, others represent the other resting brain networks such as the salience, auditory, visual resting networks, etc. Finally, other components represent noise/artifact components with no biological meaning. The components were sorted and spatially correlated with the Default Mode template provided by GIFT for DMN identification and were also visually inspected. Finally, the best-fit component of each individual (z-maps) was used to perform group statistical analyses (second level analyses).

\section{Statistical analysis}

For the group analysis, second level General Linear Model (GLM) method of the SPM8 (Statistical Parametrical Mapping, http://www.fil.ion.ucl.ac.uk) was used.

The first step was to study the global pattern of DMN activation at the group level. All the individual default mode $\mathrm{z}$ maps that were extracted in the ICA analyses described above, were included in the same group and a one-sample $t$ test ( $p<0.05 \mathrm{FWE}$ corrected for multiple comparisons, extent threshold $k=30$ voxels) was performed. The same DMN template mask that has been used to sort the components of the ICA, was applied to extract the results.

After this all the individual DMN z-maps were entered to SPM8 and a multiple regression (with positive and negative correlations) was performed $(p<0.01$ uncorrected, extent threshold $k=10$ voxels), using all subjects in the study and their respective scores in HARS and HDRS. In order to control for age and gender, both were added as covariates in the design matrix. The equations for the GLM used in the statistical analysis were:

For Anxiety $: \mathrm{y}=\beta 0+\beta 1 *$ Anxiety Score $+\beta 2 *$ Age $+\beta 3 *$ Gender $+\varepsilon$

For Depression : $\mathrm{y}=\beta 0+\beta 1 *$ Depression Score $+\beta 2 *$ Age

$$
+\beta 3 * \text { Gender }+\varepsilon
$$

The resulting statistical maps ( $\mathrm{t}$ and $\mathrm{z}$ statistics) represent the strength of the association between anxiety/depression scores and DMN functional connectivity. These results were masked with the DMN template provided by GIFT. The results were corrected for multiple comparisons using the "AlphaSim" implemented in REST (http://restfmri.net/ forum/REST_V1.8). This function is based on the Monte Carlo simulation in AFNI (http://afni.nimh.nih.gov/afni/doc/ manual/AlphaSim) (Song et al. 2011). A corrected threshold of $p<0.05$ was obtained using a combined threshold of $p<0$. 01 and a minimum cluster size of 23 voxels. Within the template, the DMN regions were reported and anatomical labeling was assigned by a combination of visual inspection and the Anatomical Automatic Labeling atlas (AAL) (Tzourio-Mazoyer et al. 2002).

\section{Results}

\section{DMN network}

The DMN was identified in the resting state conditions at the group level. The main components of the DMN were observed as can be seen in Fig. 1. The statistics of the group DMN pattern are represented in Tables 1 and 2. 

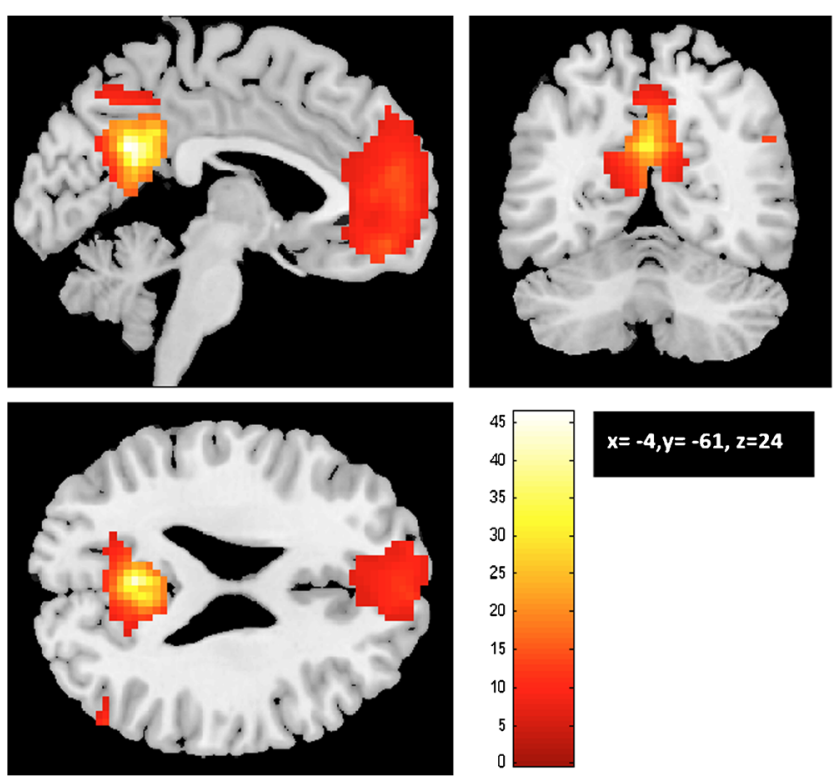

Fig. 1 Group-level spatial pattern of DMN ( $p<0.05$ FWE corrected)

\section{DMN and depression}

The regions that showed an increased functional connectivity associated with increased depression scores were the right superior orbitofrontal gyrus $(x=24, y=39, z=-12)$ and the left middle frontal gyrus $(x=-45, y=39, z=18)$.

The regions showing increased functional connectivity associated with a decrease in depression scores were the left middle occipital gyrus $(x=-27, y=-78, z=12)$, left fusiform gyrus ( $x=-21, y=-84, z=-6)$, left precuneus $(x=-9, y=-45$, $z=60)$, left middle cingulum $(x=-15, y=-39, z=42)$, left posterior cingulate cortex $(x=0, y=-36, z=30)$, left inferior parietal cortex $(x=-48, y=-48, z=60)$ and left angular gyrus $(x=-45, y=-63, z=48)$ (Fig. 2).

\section{DMN and anxiety}

The regions that showed increased functional connectivity associated with increased anxiety scores were the right superior medial frontal gyrus $(x=18, y=45, z=0)$, right medial orbitofrontal gyrus $(x=12, y=39, z=-9)$, left anterior

Table 1 DMN global functional connectivity (FWE $<0.05$ corrected)

\begin{tabular}{llll}
\hline Regions & $\mathrm{Z}$ & $\mathrm{k}$ (cluster size) & MNI coordinates $(\mathrm{x}, \mathrm{y}, \mathrm{z})$ \\
\hline Left precuneus & $>8$ & 734 & $-3,-57,27$ \\
Left medial prefrontal & 7.68 & 1417 & $0,48,-15$ \\
Right angular & 7.06 & 54 & $51,-60,30$ \\
Right middle frontal & 6.63 & 56 & $18,42,48$ \\
Right middle cingulum & 6 & 30 & $3,-21,36$ \\
Right medial temporal & 5.97 & 41 & $24,-18,-24$ \\
Left medial temporal & 5.76 & 62 & $-24,-24,-21$ \\
\hline
\end{tabular}

cingulum $(x=15, y=39, z=12)$, left middle frontal gyrus $(x=$ $-42, y=42, z=24)$, left inferior triangular gyrus $(x=-36, y=$ $30, z=24)$, right superior orbitofrontal gyrus $(x=18, y=27, z=$ $-15)$ (Table 3).

The regions showing increased functional connectivity when anxiety scores decreased were the left post-central gyrus $(x=-18, y=-36, z=81)$, left middle temporal gyrus $(x=-54$, $y=-45, z=0)$ left inferior temporal gyrus $(x=-51, y=-54, z=$ -6) (Fig. 3).

\section{Discussion}

In the present study, we aimed at analyzing the relationship between depressive and anxiety states and DMN functional connectivity (FC) in a sample of healthy subjects. Overall, our findings confirmed the hypothesis of a dissociation between anterior and posterior areas of the DMN. Specifically, we found that frontal portions of the DMN had increased FC in more anxious and depressed individuals, whereas posterior areas showed a reduced FC. These results are consistent with previous studies of DMN alterations in depression and anxiety (e.g., Greicius et al. 2007; Zhao et al. 2007; Zhu et al. 2012).

Before discussing our results, it is important to note that the current study looks at alterations of the DMN associated with the variability in anxiety and depressive states in a non-clinical sample. However, we believe that FC alterations in healthy individuals, with different levels of depression and anxiety, may be helpful in understanding subtle alterations that may increase vulnerability to anxiety and mood disorders. As Lemogne et al. (2012) argued in their meta-analysis on the role of elevated MPFC activation in depression, studies in never-depressed healthy subjects presenting with increased vulnerability for depression are warranted because some of the findings with clinical populations may result from enduring effects of acute depression or anxiety rather than from premorbid vulnerability. Therefore, resting state neuroimaging assessment in healthy individuals presenting with a natural variation in depression or anxiety symptoms, may have an important role in predicting the course of affective disorders. Specifically, it can help us to predict when normal mood alterations may lead to the emergence of clinical depression or anxiety. For example the heightened FC at rest in anterior portions of the DMN in normal depressive and anxiety states found by us, may constitute a predisposing factor for overresponsiveness of these regions to negative stimuli, contributing to the onset of a depressive or anxiety disorder.

In fact, the anterior- posterior DMN dissociation observed in our sample has also been demonstrated in clinically depressed and anxious subjects who present higher connectivity in structures such as the orbitofrontal and middle frontal cortex, and lower connectivity in the PCC, precuneus, inferior parietal, angular and occipital gyrus (Zhu et al. 2012). 
Table 2 Correlations between depression scores and DMN functional connectivity ${ }^{\text {a }}$

\begin{tabular}{lllll}
\hline Brain region & $T$ value & $Z$ value & $\mathrm{k}$ (cluster size) & MNI coordinates (x,y,z) \\
\hline $\begin{array}{l}\text { Positive correlations } \\
\quad \text { Right superior orbitofrontal gyrus }\end{array}$ & 4.34 & 3.60 & 76 & \\
$\quad 3.25$ & 2.87 & 39 & $24,39,-12$ \\
$\quad$ Left middle frontal gyrus & & & & $-45,39,18$ \\
Negative correlations & 4.30 & 3.57 & 87 & $-27,-78,12$ \\
Left middle occipital gyrus & 3.70 & 3.19 & & $-21,-84,-6$ \\
$\quad$ Left fusiform gyrus & 4.01 & 3.40 & 31 & $-9,-45,60$ \\
$\quad$ Left precuneus & 3.94 & 3.35 & 56 & $-15,-39,42$ \\
$\quad$ Left middle cingulum & 2.78 & 2.53 & & $-48,-48,60$ \\
$\quad$ Left posterior cingulate cortex & 3.23 & 2.86 & 43 & $-39,-48,48$ \\
$\quad$ Left inferior parietal gyrus & 3.04 & 2.72 & & \\
$\quad$ Left angular gyrus & & & & \\
\hline
\end{tabular}

${ }^{a}$ Each cluster's coordinates in Montreal Neurological Institute (MNI) space (x, y, z), maximum T and Z-value and size are given. All correlations were significant at a corrected for multiple comparisons $p<0.05$ threshold (based on the combination of a threshold of $p<0.01$ and a minimum cluster size of 23 voxels), determined by Monte Carlo simulation program (AlphaSim)
Connectivity analysis allows us to explore whether the altered DMN pattern observed in depression and anxiety is disproportionately driven by the activity of specific nodes (e.g., anterior versus posterior nodes). On the other hand, FC studies may contribute to integrate findings from studies reporting specific regions of hyper metabolism in certain disorders, into the broader context of an altered neural network such as the DMN.

In fact, several task positive or activation studies at rest have found that depressed individuals present an increased metabolism in several midline anterior regions including medial prefrontal, anterior cingulate and orbitofrontal cortex (e. g

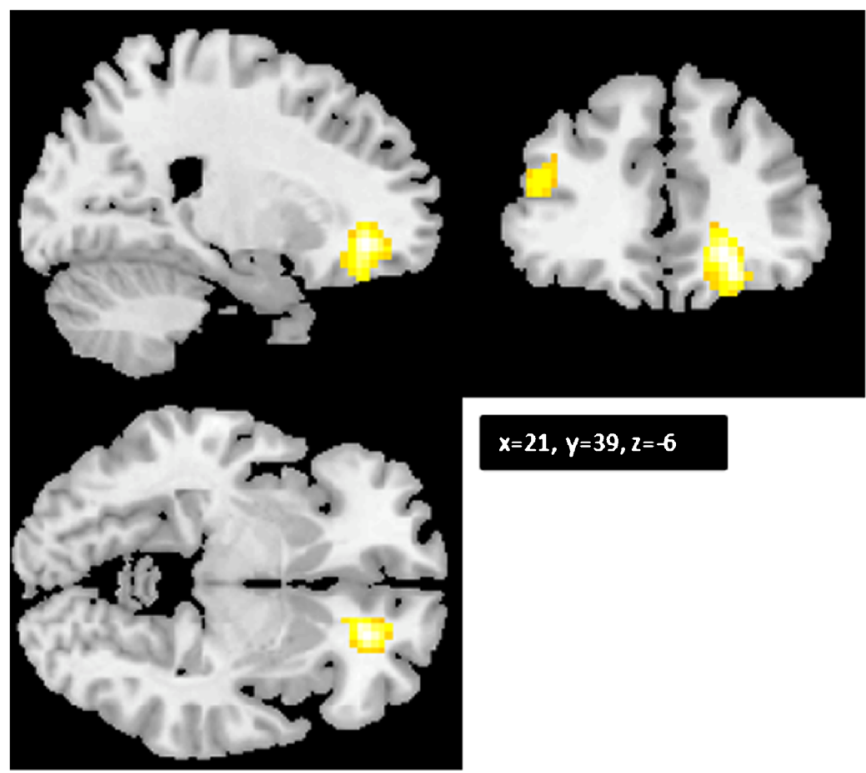

Fig. 2 DMN regions positively (warm colors) and negatively (cool colors) correlated with depression scores. The image on the left shows the right superior orbitofrontal gyrus and left middle frontal gyrus. The
Beauregard et al. 2006). This is not surprising considering the role of frontal midline areas for self-referential processing (Northoff et al. 2006) and rumination typically experienced by individuals who report depressive symptoms (Berman et al. 2011; Grimm et al. 2011). These findings are also consistent with previous evidence for an increased MPFC activation for negative stimuli in individuals with high scores of neuroticism or dispositional self-focus (Haas et al., 2008; Cremers et al. 2010; Eisenberger et al., 2005), which are a proxy of vulnerability for depression.

The same is true for anxiety in which the same frontal DMN areas that we found that were positively correlated

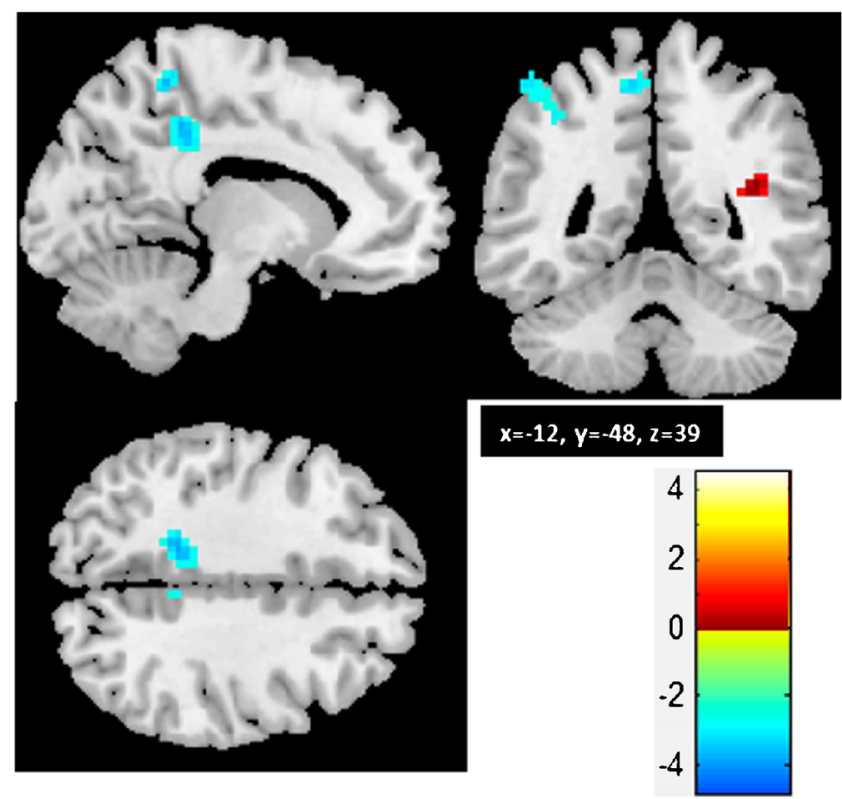

image on the right shows the left posterior cingulate cortex, left precuneus and left angular gyrus 
Table 3 Correlations between anxiety scores and DMN functional connectivity ${ }^{\text {a }}$

\begin{tabular}{lllll}
\hline Brain regions & $T$ & $Z$ & $\mathrm{k}$ (cluster size) & MNI Coordinates (x,y,z) \\
\hline Positive correlations & & & & \\
$\quad$ Right medial prefrontal gyrus & 4.59 & 3.75 & 60 & $18,45,0$ \\
$\quad$ Right medial orbitofrontal gyrus & 3.54 & 3.08 & & $12,39,-9$ \\
$\quad$ Right anterior cingulate gyrus & 3.06 & 2.74 & & $15,39,12$ \\
$\quad$ Left middle frontal gyrus & 3.70 & 3.19 & 51 & $-42,42,24$ \\
$\quad$ Left inferior triangular gyrus & 2.95 & 2.66 & & $-36,30,24$ \\
$\quad$ Right superior orbitofrontal gyrus & 3.20 & 2.84 & 36 & $18,27,-15$ \\
Negative correlations & & & & $-18,-36,81$ \\
$\quad$ Left post central gyrus & 4.45 & 3.67 & 43 & $-54,-45,0$ \\
$\quad$ Left middle temporal gyrus & 3.57 & 3.10 & 33 & $-51,-54,-6$ \\
$\quad$ Left inferior temporal gyrus & 3.40 & 2.98 & & \\
\hline
\end{tabular}

${ }^{a}$ Each cluster's coordinates in Montreal Neurological Institute (MNI) space (x, y, z), maximum T and Z-value and size are given. All correlations were significant at a corrected for multiple comparisons $p<0.05$ threshold (based on the combination of a threshold of $p<0.01$ and a minimum cluster size of 23 voxels), determined by Monte Carlo simulation program (AlphaSim) with anxiety (MPFC, ACC, middle frontal gyrus and $\mathrm{OBF}$ ), were previously reported to be overactive during anxious cognition (Gusnard et al. 2001; Milad and Rauch 2007). This is in line with the well documented role of the MPFC and OBF in the regulation of emotional arousal in general (e.g., Etkin et al. 2006), and specifically in anxiety related processing (Qin and Northoff 2011; Ray et al. 2005) and with the increased activity of the MPFC and OBF in several anxiety disorders (Fredrikson et al., 1995; Shin et al. 2013).

The FC of the anterior cingulate cortex was positively correlated with anxiety scores. This area has been positively

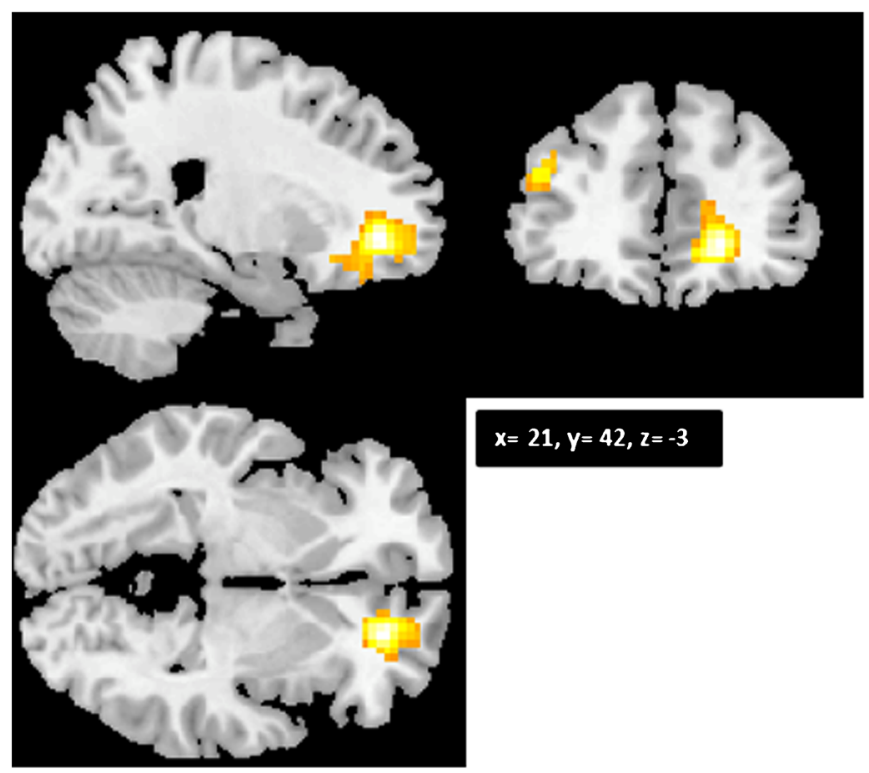

Fig. 3 DMN regions positively (warm colors) and negatively (cool colors) correlated with anxiety scores. The image on the left shows the right medial orbitofrontal gyrus and adjacent anterior cingulate cortex, associated with anxiety states both in healthy individuals (Chua et al. 1999; Kimbrell et al. 1999; Osuch et al. 2000) and in patients with disorders such as social anxiety (Liao et al. 2010) and panic disorder (Shin et al. 2013). Given that the ACC is involved in affective processing of negative information (Amir et al. 2005), being especially sensitive to internal signs of autonomic arousal (Shin et al. 2013), it is not surprising that more hyper vigilant and alert participants presented higher FC in this area. This was the only frontal DMN area differentiating anxiety from depression. In fact, we did not replicate the findings reporting increased activity in the subgenual ACC in depression (e.g., Mayberg 1997; Drevets

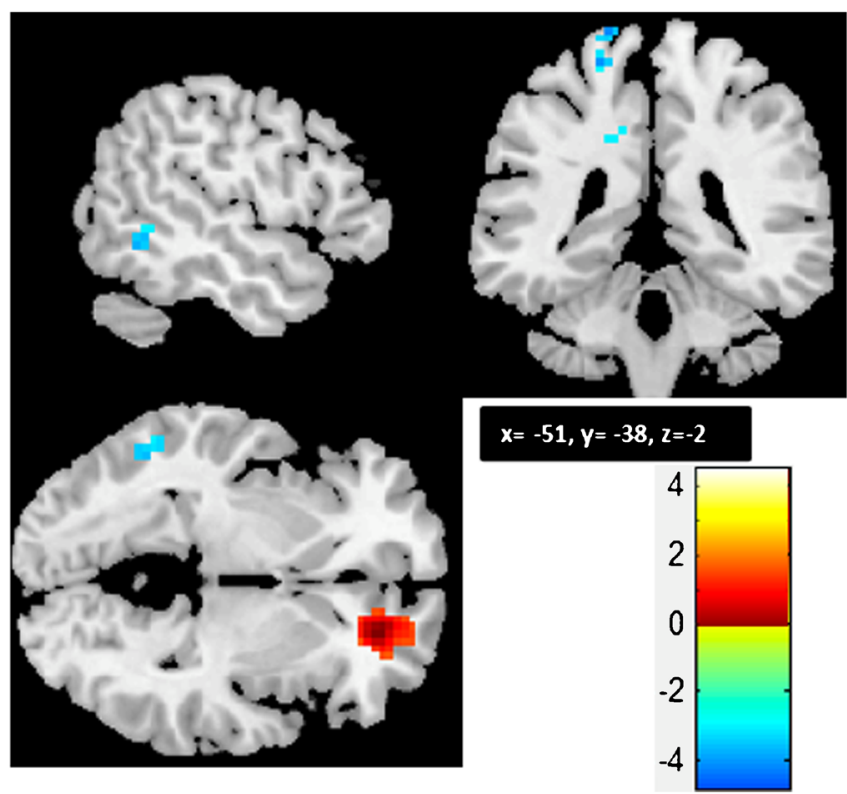

and the left middle frontal gyrus. The image on the right shows the left post central and left temporal areas 
et al. 2008; Kennedy et al. 2001). It has been shown that the over activation in the ACC in depression tends to decline towards the normal range in depressed patients who respond to treatment (e.g., Kimbrell et al. 1999). Thus, the increased activation of the ACC may be a defining characteristic of severe/clinically significant symptoms of depression and not of normative depressive states.

On the contrary, posterior DMN areas, such as the PCC, precuneus, angular gyrus, and inferior parietal cortex presented decreased FC in more depressed and anxious individuals. Our findings of a negative correlation between the FC of posterior DMN areas and anxiety can work as a cross-modal validation for task positive studies linking these areas to functions that are compromised in depressive states. In fact, these posterior DMN areas have been associated with autobiographical memory (Olson et al. 2007; Spreng and Grady 2010), social cognition (Vogeley and Fink 2003), and visual and sensory processing (Ganis et al. 2004), and may thus be related with the episodic memory impairments typically reported in depressed patients (Lemogne et al. 2006, 2009; Kuyken and Howell 2006), namely the over-general autobiographical memory phenomena (Greicius et al. 2007; Zhu et al. 2012).

Regarding anxiety, we also found a negative correlation with posterior DMN areas. Interestingly, the areas whose FC were negatively associated with higher scores were the post central gyrus and in the middle and inferior temporal gyrus. These results may suggest that there are different mechanisms associated with anxiety states versus depressive states. Support for a specific involvement of these two areas in anxiety comes from findings by Kimbrell et al. (1999) of a decreased activity in posterior temporo-parietal cortex with anxiety induction. The ITG is involved in visual perception (Ishai et al. 1999) and is part of the ventral visual pathway (Baddeley et al. 1997), suggesting that the decreased activity in sensory processing areas may represent a neural correlate of perceptual impairments in anxious patients (Liao et al. 2010).

In conclusion, we found that more depressed and anxious individuals presented an over connectivity of frontal DMN areas during the brain's resting state. This altered baseline connectivity of the frontal DMN may be related to the continuous rumination and anxiety-related thoughts focused on the perception of loss, threat, and injustice (Takano and Tanno 2009), allocating excessive cognitive resources to selfreferential processes at the cost of externally oriented processing such as problem-solving (Harvey et al. 2005). Thus, our results bring additional support for the role for self-focus in depression and the anterior DMN nodes in its pathophysiology.

On the contrary, the posterior nodes of the DMN such as the PCC and temporal areas seem to be more involved in tasks such as autobiographic episodic memory and performing episodic judgments about the future (Behrmann et al. 2004; DeYoung et al. 2010). Thus, our study provided additional evidence for a dissociation between anterior and posterior midline regions that compose the DMN, raising the possibility of a functional specialization within the DMN in terms of selfreferential tasks. The idea that midline areas concerned with self-referential processing may be domain or content specific was suggested in the meta-analysis by Northoff et al. (2006). The anterior regions strongly connected with limbic areas are involved in the emotional self-referential processing (Ochsner and Gross 2005) and representing interoceptive stimuli as selfreferential (d'Argembeau et al. 2005). On the contrary, the posterior regions densely connected with the hippocampus are more implicated in coding and retrieving autobiographical memory, being responsible to putting self-referential stimuli within a temporal context linking them to past self-referential events.

A potential limitation of our study is the fact that only the pattern of the DMN at rest was analyzed. Several authors (Sridharan et al. 2008; Hamilton et al. 2011; WhitfieldGabrieli and Ford 2012) pointed out the importance of examining the dynamics of activation- deactivation of the DMN, during the transition from rest to task performance. Futures studies should overcome this limitation by analyzing the patterns of DMN activation and deactivation in depressive and anxiety states.

Acknowledgments This research was funded by the Portuguese Foundation for Science and Technology (FCT): PIC/IC/83290/2007, which is supported by FEDER (POFC - COMPETE). Joana Coutinho was funded by a FCT postdoctoral grant (number: SFRH/BPD/75014/2010)- POPH program and Bial Foundation (grant number 87/12)

Liliana Maia is supported by the Competitive Factors Operational Programme-COMPETE_- by national funds from the Portuguese Foundation for Science and Technology (grant PTDC/PSI-PCL/115316/2009).

Conflict of interest Joana Coutinho, Sara Fernandes, José Miguel Soares, Liliana Maia, Óscar F. Gonçalves and Adriana Sampaio declare that they have no conflict of interest.

Ethical standards All procedures followed were in accordance with the ethical standards of the responsible committee on human experimentation (institutional and national) and with the Helsinki Declaration of 1975, and the applicable revisions at the time of the investigation. Informed consent was obtained from all patients for being included in the study.

\section{References}

Amir, N., Klumpp, H., Elias, J., Bedwell, J. S., Yanasak, N., \& Miller, L. S. (2005). Increased activation of the anterior cingulate cortex during processing of disgust faces in individuals with social phobia. Biological Psychiatry, 57, 975-981.

APA (Ed.). (2013). Diagnostic and statistical manual of mental disorders $D S M-V$ (5th ed.). Arlington: American Psychiatric Publishing. 
Baddeley, R., Abbott, L. F., Booth, M. C., Sengpiel, F., Freeman, T., Wakeman, E. A., \& Rolls, E. T. (1997). Responses of neurons in primary and inferior temporal visual cortices to natural scenes. Proceeding Biological Science, 264, 1775-1783.

Bartels, A., \& Zeki, S. (2005). Brain dynamics during natural viewing conditios - a new guide for maping connectivity in vivo. NeuroImage, 24, 339-349.

Beauregard, M., Paquette, V., \& Levesque, J. (2006). Dysfunction in the neural circuitry of emotional self-regulation in major depressive disorder. NeuroReport, 17, 843-846.

Beck, A. T. (Ed.). (1976). Cognitive therapy and the emotional disorders. New York: International University Press.

Beckmann, C. F., DeLuca, M., Devlin, J. T., \& Smith, S. M. (2005). Investigations into resting-state connectivity using independent component analysis. Philosophical Transactions of the Royal Society of London. Series B, Biological Sciences, 360(1457), 1001-1013.

Behrmann, M., Geng, J. J., \& Shomstein, S. (2004). Parietal cortex and attention. Current Opinion in Neurobiology, 14(2), 212-217.

Berman, M. G., Peltier, S., Nee, D. E., Kross, E., Deldin, P. J., \& Jonides, J. (2011). Depression, rumination and the default network. Social Cognitive and Affective Neuroscience, 6(5), 548-555.

Buckner, R. L., Andrews-Hanna, J. R., \& Schacter, D. L. (2008). The brain's default network: anatomy, function, and relevance to disease. Annals of the New York Academy of Sciences, 1124, 1-38.

Buckholtz \& Meyer-Lindenberg (2012). Psychopathology and the Human Connectome: Toward a Transdiagnostic Model of Risk For Mental Illness. Neuron 74

Calhoun, V. D., Eichele, T., \& Pearlson, G. (2009). Functional brain networks in schizophrenia: a review. Frontiers Human Neuroscience, 3, 17.

Cameron, O. G., Zubieta, J. K., Grunhaus, L., \& Minoshima, S. (2000). Effects of yohimbine on cerebral blood flow, symptoms, and physiological functions in humans. Psychosomatic Medicine, 62, 549559.

Chao-Gan, Y., \& Yu-Feng, Z. (2010). DPARSF: a MATLAB toolbox for "Pipeline" data analysis of resting-state fMRI. Frontiers System Neuroscience, 4, 13.

Chua, P., Krams, M., Toni, I., Passingham, R., \& Dolan, R. (1999). A functional anatomy of anticipatory anxiety. NeuroImage, 9, 563571.

Clark, D. M., \& Wells, A. (1995). A cognitive model of social phobia. In R. G. Heimberg, M. R. Liebowitz, D. Hope, \& F. Schneider (Eds.), Social phobia: Diagnosis, assessment and treatment (pp. 69-93). New York: Guildford.

Correa, N., Adali, T., Li, Y., \& Calhoun, V. (2005). Comparison of blind source separation algorithms for FMRI using a new Matlab toolbox: GIFT. Proceedings of the IEEE International Conference Acoustics, Speech, and Signal Processing, 5, 401-404.

Cremers, H. R., Demenescu, L. R., Aleman, A., Renken, R., van Tol, M. J., van der Wee, N. J., Veltman, D. J. \& Roelofs, K. (2010) Neuroticism modulates amygdala-prefrontal connectivity in response to negative emotional facial expressions. Neuroimage 49, 963-970.

d'Argembeau, A., Collette, F., Van der Linden, M., Laureys, S., Del Fiore, G., Degueldre, C., Luxen, A., \& Salmon, E. (2005). Selfreferential reflective activity and its relationship with rest: a PET study. NeuroImage, 25, 616-624.

DeYoung, C. G., Hirsh, J. B., Shane, M. S., Papademetris, X., Rajeevan, N., \& Gray, J. R. (2010). Testing predictions from personality neuroscience. Psychological Science, 21(6), 820.

Drevets, W. C., Price, J. L., \& Furey, M. L. (2008). Brain structural and functional abnormalities in mood disorders: implications for neurocircuitry models of depression. Brain Structure and Function, 213, 93-118.
Etkin, A., Egner, T., Peraza, D. M., Kandel, E. R., \& Hirsch, J. (2006). Resolving emotional conflict: a role for the rostral anterior cingulate cortex in modulating activity in the amygdala. Neuron, 51, 871-882.

Eisenberger, N. I., Lieberman, M. D., Satpute, A. B. (2005). Personality from a controlled processing perspective: an fMRI study of neuroticism, extraversion, and self-consciousness. Cognitive Affective and Behavior Neuroscience 5, 169-181

Fox, M. D., \& Raichle, M. E. (2007). Spontaneous fluctuations in brain activity observed with functional magnetic resonance imaging. Nature Review Neuroscience, 8(9), 700-711.

Fredrikson, M., Wik ,G., Annas, P., Ericson, K., \& Stone-Elander, S, (1995). Functional neuroanatomy of visually elicited simple phobic fear: Additional data and theoretical analysis. Psychophysiology 32: 43- 48 .

Ganis, G., Thompson, W. L., \& Kosslyn, S. M. (2004). Brain areas underlying visual mental imagery and visual perception: an fMRI study. Brain Research. Cognitive Brain Research, 20(2), 226-241.

Garrity, A. G., Pearlson, G. D., McKiernan, K., Lloyd, D., Kiehl, K. A., \& Calhoun, V. D. (2007). Aberrant "default mode" functional connectivity in schizophrenia. American Journal of Psychiatry, 164(3), 450-457.

Gorman, J. M. (1996). Comorbid depression and anxiety spectrum disorders. Depression and Anxiety, 4(4), 160-168.

Greicius, M. D., Krasnow, B., Reiss, A. L., \& Menon, V. (2003). Functional connectivity in the resting brain: a network analysis of the default mode hypothesis. Proceedings of the National Academy of Sciences of the United States of America, 98, 4259-4264.

Greicius, M. D., Flores, B. H., Menon, V., Glover, G. H., Solvason, H. B., Kenna, H., et al. (2007). Resting-state functional connectivity in major depression: abnormally increased contributions from subgenual cingulate cortex and thalamus. Biological Psychiatry, 62(5), 429-437.

Grimm, S., Boesiger, P., Beck, J., Schuepbach, D., Bermpohl, F., Walter, M., et al. (2009). Altered negative BOLD responses in the defaultmode network during emotion processing in depressed subjects. Neuropsychopharmacology, 34, 932-943.

Grimm, S., Ernst, J., Boesiger, P., Schuepbach, D., Boeker, H., \& Northoff, G. (2011). Reduced negative BOLD responses in the default-mode network and increased self-focus in depression. World Journal of Biological Psychiatry, 12(8), 627-637.

Gusnard, D. A., Raichle, M. E., \& Raichle, M. E. (2001). Searching for a baseline: functional imaging and the resting human brain. Nature Review Neuroscience, 2(10), 685-694.

Haas, B. W., Constable, R. T. \& Canli, T. (2008). Stop the sadness: neuroticism is associated with sustained medial prefrontal cortex response to emotional facial expressions. Neuroimage 42, 385-392.

Hamilton, M. (1959). The assessment of anxiety states by rating. British Journal of Medical Psychology, 32(1), 50-55.

Hamilton, M. (1967). Development of a rating scale for primary depressive illness. British Journal of Social \& Clinical Psychology, 6(4), 278-296.

Hamilton, J. P., Furman, D. J., Chang, C., Thomason, M. E., Dennis, E., \& Gotlib, I. H. (2011). Default-mode and task-positive network activity in major depressive disorder: implications for adaptive and maladaptive rumination. Biological Psychiatry, 70(4), 327-333.

Harvey, P. O., Fossati, P., Pochon, J. B., Levy, R., Lebastard, G., Lehericy, S., et al. (2005). Cognitive control and brain resources in major depression: an fMRI study using the n-back task. NeuroImage, 26, $860-869$

Himberg, J., Hyvärinen, A., \& Esposito, F. (2004). Validating the independent components of neuroimaging time series via clustering and visualization. NeuroImage, 22(3), 1214-1222.

Insel T., Cuthbert B., Garvey M., Heinssen, R., Pine D. S., Quinn, K., Sanislow C. \& Wang P. (2010). Research Domain Criteria (RDoC): 
Toward a New Classification Framework for Research on Mental Disorders. American Journal of Psychiatry 167:7, 748-751.

Ishai, A., Ungerleider, L. G., Martin, A., Schouten, J. L., \& Haxby, J. V. (1999). Distributed representation of objects in the human ventral visual pathway. Proceedings of the National academy of Sciences of the United States of America, 96, 9379-9384.

Kennedy, D. P., \& Courchesne, E. (2008). The intrinsic functional organisation of the brain is altered in autism. NeuroImage, 39, 1877 1885.

Kennedy, S. H., Evans, K. R., Kruger, S., Mayberg, H. S., Meyer, J. H., McCann, S., et al. (2001). Changes in regional brain glucose metabolism measured with positron emission tomography after paroxetine treatment of major depression. American Journal of Psychiatry, 158, 899-905.

Kimbrell, T. A., George, M. S., Parekh, P. I., Ketter, T. A., Podell, D. M., Danielson, A. L., et al. (1999). Regional brain activity during transient self-induced anxiety and anger in healthy adults. Biological Psychiatry, 46, 454-465.

Kross, E., Davidson, M., Weber, J., \& Ochsner, K. (2009). Coping with emotions past: the neural bases of regulating affect associated with negative autobiographical memories. Biological Psychiatry, 65, 361-366.

Kuyken, W., \& Howell, R. (2006). Facets of autobiographical memory in adolescents with major depressive disorder and never depressed controls. Cognitive Emotions, 20, 466-487.

Lemogne, C., Piolino, P., Friszer, S., Claret, A., Girault, N., Jouvent, R., Allilaire, J. F., \& Fossati, P. (2006). Episodic autobiographical memory in depression: specificity, autonoetic consciousness and self-perspective. Consciousness and Cognition, 15, 258-268.

Lemogne, C., le Bastard, G., Mayberg, H., Volle, E., Bergouignan, L., Lehericy, S., et al. (2009). In search of the depressive self: extended medial prefrontal network during self-referential processing in major depression. Social Cognitive and Affective Neuroscience, 4(3), 305312.

Lemogne, C., Delaveau, P., Freton, M., Guionnet, S., \& Fossati, P. (2012) Medial prefrontal cortex and the self in major depression. Journal of Affective Disorders, 136

Liao, W., Chen, H., Feng, Y., Mantini, D., Gentili, C., Pan, Z., et al. (2010). Selective aberrant functional connectivity of resting state networks in social anxiety disorder. NeuroImage, 52(4), 1549-1558.

Mayberg, H. S. (1997). Limbic-cortical dysregulation: a proposed model of depression. Journal of Neuropsychiatry and Clinical Neurosciences, 9(3), 471-481.

Milad, M. R., \& Rauch, S. L. (2007). The role of the orbitofrontal cortex in anxiety disorders. Annals of the New York Academy of Sciences, $1121,546-561$.

Mor, N., \& Winquist, J. (2002). Self-focused attention and negative affect: a meta-analysis. Psychological Bulletin, 128(4), 638-662.

Norman, S., Bedirhan, U. T., Yves, L., \& Hans-Ulrich, W. (1996). Depression comorbid with anxiety: results from the WHO study on psychological disorders in primary health care. The British Journal of Psychiatry, 168(30), 38-43.

Northoff, G., Heinzel, A., de Greck, M., Bermpohl, F., Dobrowolny, H., \& Panksepp, J. (2006). Self-referential processing in our brain-a meta-analysis of imaging studies on the self. NeuroImage, 31(1), 440-457.

Ochsner, K. N., \& Gross, J. J. (2005). The cognitive control of emotion. Trends in Cognitive Sciences, 9(5), 242-249.

Oldfield, R. C. (1971). The assessment and analysis of handedness: the Edinburgh inventory. Neuropsychologia, 9(1), 97-113.

Olson, I. R., Plotzker, A., \& Ezzyat, Y. (2007). The Enigmatic temporal pole: a review of findings on social and emotional processing. Brain, 130(Pt 7), 1718-1731.

Öngür, D., Lundy, M., Greenhouse, I., Shinn, A. K., Menon, V., Cohen, B. M., et al. (2010). Default mode network abnormalities in bipolar disorder and schizophrenia. Psychiatry Research: Neuroimaging, 183(1), 59-68.

Osuch, E. A., Ketter, T. A., Kimbrell, T. A., George, M. S., Benson, B. E., Willis, M. W., et al. (2000). Regional cerebral metabolism associated with anxiety symptoms in affective disorder patients. Biological Psychiatry, 48(10), 1020-1023.

Pignat, J. M., Koval, O., Van De Ville, D., Voloshynovskiy, S., Michel, G., \& Pun, T. (2013). The impact of denoising on independent component analysis of functional magnetic resonance imaging data. Journal of Neuroscience Methods, 213(1), 105-122.

Qin, P., \& Northoff, G. (2011). How is our self related to midline regions and the default-mode network? NeuroImage, 57(3), 1221-1233.

Raichle, M. E., MacLeod, A. M., Snyder, A. Z., Powers, W. J., Gusnard, D. A., \& Shulman, G. L. (2001). A default mode of brain function. Proceedings of the National Academy of Sciences, 98, 676-682.

Ray, R. D., Ochsner, K. N., Cooper, J. C., Robertson, E. R., Gabrieli, J. D., \& Gross, J. J. (2005). Individual differences in trait rumination and the neural systems supporting cognitive reappraisal. Cognitive, Affective, \& Behavioral Neuroscience, 5(2), 156-168.

Sampaio, A., Soares, J. M., Coutinho, J., Sousa, N., \& Goncalves, O. F. (2013). The big five default brain: functional evidence. Brain Structure and Function.

Shin, Y. W., Dzemidzic, M., Jo, H. J., Long, Z., Medlock, C., Dydak, U., et al. (2013). Increased resting-state functional connectivity between the anterior cingulate cortex and the precuneus in panic disorder: resting-state connectivity in panic disorder. Journal of Affective Disorders, 150(3), 1091-1095.

Song, X. W., Dong, Z. Y., Long, X. Y., Li, S. F., Zuo, X. N., Zhu, C. Z., et al. (2011). REST: a toolkit for resting-state functional magnetic resonance imaging data processing. PLoS One, 6(9), e25031.

Sorg, C., Riedl, V., Muhlau, M., Calhoun, V. D., Eichele, T., Laer, L., et al. (2007). Selective changes of resting-state networks in individuals at risk for Alzheimer's disease. Proceedings of the National Academy of Sciences of the United State of America, 104(47), 18760-18765.

Spreng, R. N., \& Grady, C. L. (2010). Patterns of brain activity supporting autobiographical memory, prospection, and theory of mind, and their relationship to the default mode network. Journal of Cognitive Neuroscience, 22(6), 1112-1123.

Sridharan, D., Levitin, D. J., \& Menon, V. (2008). A critical role for the right fronto-insular cortex in switching between central-executive and default- mode networks. Proceedings of the National Academy of Sciences of the United States of America, 105, 1256912574.

Takano, K., \& Tanno, Y. (2009). Self-rumination, self-reflection, and depression: self-rumination counteracts the adaptive effect of selfreflection. Behavioral Research Therapy, 47(3), 260-264.

Tzourio-Mazoyer, N., Landeau, B., Papathanassiou, D., Crivello, F., Etard, O., Delcroix, N., et al. (2002). Automated anatomical labeling of activations in SPM using a macroscopic anatomical parcellation of the MNI MRI single-subject brain. NeuroImage, 15(1), 273-289.

Uddin, L. Q., Kelly, A. M., Biswal, B. B., Margulies, D. S., Shehzad, Z., Shaw, D., et al. (2008). Network homogeneity reveals decreased integrity of default-mode network in ADHD. Journal of Neuroscience Methods, 169(1), 249-254.

Vogeley, K., \& Fink, G. R. (2003). Neural correlates of the first-personperspective. Trends Cognitive Science, 7(1), 38-42.

Wang, K., Jiang, T., Yu, C., Tian, L., Li, J., Liu, Y., et al. (2008). Spontaneous activity associated with primary visual cortex: a resting-state FMRI study. Cerebral Cortex, 18(3), 697-704.

Watkins, E. R. (2008). Constructive and unconstructive repetitive thought. Psychological Bulletin, 134(2), 163-206.

Whitfield-Gabrieli, S., \& Ford, J. M. (2012). Default mode network activity and connectivity in psychopathology. Annual Review Clinical Psychology, 8, 49-76. 
Zhao, X. H., Wang, P. J., Li, C. B., Hu, Z. H., Xi, Q., Wu, W. Y., et al. (2007). Altered default mode network activity in patient with anxiety disorders: an fMRI study. European Journal of Radiology, 63(3), 373-378.

Zhou, Y., Liang, M., Tian, L., Wang, K., Hao, Y., Liu, H., et al. (2007). Functional disintegration in paranoid schizophrenia using restingstate fMRI. Schizophrenia Research, 97, 194-205.
Zhou, Y., Yu, C., Zheng, H., Liu, Y., Song, M., Qin, W., et al. (2010). Increased neural resources recruitment in the intrinsic organization in major depression. Journal of Affective Disorders, 121, 220-230.

Zhu, X., Wang, X., Xiao, J., Liao, J., Zhong, M., Wang, W., et al. (2012). Evidence of a dissociation pattern in resting-state default mode network connectivity in first-episode, treatment-naive major depression patients. Biological Psychiatry, 71(7), 611-617. 